\title{
EPOC
}

\section{El reflujo gastroesofágico es un factor de riesgo de exacerbaciones de enfermedad pulmonar obstructiva crónica}

\section{Gastroesophageal reflux is a risk factor for exacerbations of chronic obstructive lung disease}

Nathali Moreno Cortés ${ }^{(1)}$; Carlos Celis Preciado, MD(2)

\footnotetext{
(1) Interna, Pontificia Universidad Javeriana, Hospital Universitario San Ignacio, Bogotá, Colombia.

${ }^{(2)}$ Internista, Neumólogo, Hospital Universitario San Ignacio, Pontificia Universidad Javeriana, Bogotá, Colombia.

Correspondencia: Carlos Celis Preciado. Correo electrónico: cacelis.neumo@gmail. com

Recibido: 22-04-2013. Aceptado: 10-06-2013.
}

\section{Referencia}

Sakae TM, Pizzichini MMM, Teixeira PJZ, Silva RM, Trevisol DJ, Pizzichini E. Exacerbations of COPD and symptoms of gastroesophageal reflux: a systematic review and meta-analysis. J Bras Pneumol. 2013;39:259-271.

\section{Pregunta}

En pacientes con enfermedad pulmonar obstructiva crónica (EPOC), ¿existe relación entre los síntomas y/o el diagnóstico de reflujo gastroesofágico (RGE) y el riesgo de exacerbación?

\section{Diseño}

Revisión sistemática con metaanálisis.

Búsqueda en cuatro bases de datos electrónicas (Medline/PubMed, EMBASE, Cochrane Controlled Trials Register y LILACS) hasta el año 2012, y de las referencias bibliográficas de todos los artículos escogidos.

Se escogieron estudios en inglés, español o portugués que incluyeran pacientes con EPOC, según los criterios de la guía GOLD, enfisema o bronquitis crónica.

Dos personas revisaron todos los títulos de manera independiente, y en caso de desacuerdo se recurrió a un tercero.

\section{Estudios seleccionados}

De un total de 543 artículos que arrojó la búsqueda, se escogieron siete estudios (una cohorte prospectiva, cuatro de casos y controles, dos de corte transversal), con 2.769 pacientes, con edad promedio de 65 años y con un rango de seguimiento de dos meses a tres años.

No se evaluó formalmente la calidad metodológica de los estudios. Se evaluó la heterogeneidad y sesgo de publicación, sin presencia de alguno en los resultados..

Cuando se encontró heterogeneidad se utilizó un modelo de efectos aleatorios. Se realizó un análisis de sensibilidad mediante la comparación del modelo de efectos aleatorios y de efectos fijos. 


\section{Resultados}

\begin{tabular}{|l|l|c|}
\hline \multicolumn{1}{|c|}{ Desenlace } & \multicolumn{1}{|c|}{ Riesgo } & $\mathbf{n}$ \\
\hline $\begin{array}{l}\text { Riesgo de } \\
\text { exacerbaciones }\end{array}$ & $\begin{array}{l}\text { RR: 7,57 } \\
\text { (IC95\%: 3,84-14,94; } p \\
<0,0001)\end{array}$ & 341 \\
\hline $\begin{array}{l}\text { Número de } \\
\text { exacerbaciones por año }\end{array}$ & $\begin{array}{l}\text { MD: 0,79 } \\
(I C 95 \% \text { 0,22-1,36; } p<0,007)\end{array}$ & 2.418 \\
\hline $\begin{array}{l}\text { Prevalencia de RGE en } \\
\text { pacientes con EPOC }\end{array}$ & $\begin{array}{l}\text { RR: 13,06 } \\
(I C 95 \% 3,64-46,87 ; p<0,001)\end{array}$ & 476 \\
\hline
\end{tabular}

\section{Conclusión}

El RGE es un factor de riesgo de exacerbación de EPOC y su papel merece ser estudiado con más detalle.

\section{Comentarios}

Este metaanálisis aborda la importancia de reconocer el RGE como factor de riesgo para presentar exacerbaciones de la EPOC, por el hecho de aumentar la inflamación de las vías respiratorias, y aún más en aquellos pacientes en los que coexiste historia de bronquitis crónica. Sus objetivos están bien definidos en cuanto al tipo de estudios considerados para su inclusión y en cuanto a la metodología de búsqueda de los documentos primarios, la cual fue exhaustiva, con doble evaluación independiente, resultando homogéneo el resultado final (1).

El escaso reconocimiento del reflujo a la vía aérea como la entidad clínica causante de las exacerbaciones pulmonares, continúa en estudio; sin embargo, existen reportes de prevalencia de RGE de aproximadamente $27 \%$ en pacientes con EPOC, de ahí que se concluya que es una comorbilidad frecuente $(2,3)$.

Por lo tanto, es preciso reconocer los síntomas del RGE en todos los pacientes que presenten
EPOC ya que estarán en mayor riesgo de exacerbaciones futuras (4).

La detección del RGE en este tipo de pacientes mediante nuevas técnicas más objetivas como la determinación de pepsina en las secreciones respiratorias, podría evitar futuras hospitalizaciones y mejorar la calidad de vida al prevenir estas situaciones por medio de manejo con inhibidores de la bomba de protones, como se ha reportado en un estudio (5).

Conviene realizar más estudios en pacientes con diagnóstico de EPOC, en los que compare el papel del RGE diagnosticado por métodos como la $\mathrm{pH}$ metría (diagnóstico más objetivo) y no únicamente con base en síntomas referidos por el paciente, a fin de establecer estrategias para controlarlo y en consecuencia, disminuir el número de exacerbaciones de EPOC, hecho que conlleva a altos costos en atención y manejo en el servicio de urgencias.

\section{Conflictos de intereses}

Los autores declaran no tener conflictos de interés.

\section{Bibliografía}

1. Sakae TM, Pizzichini MM, Teixeira PJ, Silva RM, Trevisol DJ, Pizzichini E. Exacerbations of COPD and symptoms of gastroesophageal reflux: a systematic review and meta-analysis. J Bras Pneumol. 2013;39:259-271.

2. Terada K, Muro S, Sato S, Ohara T, Haruna A, Marumo S, et al. Impact of gastro-oesophageal reflux disease symptoms on COPD exacerbation. Thorax. 2008;63:951-5.

3. Hurst JR, Vestbo J, Anzueto A, Locantore N, Müllerova H, Tal-Singer $\mathrm{R}$, et al. Susceptibility to exacerbation in chronic obstructive pulmonary disease. N Engl J Med. 2010;363:1128-38.

4. Jadwiga A. Wedzicha. Is gastro-oesophageal reflux associated with COPD exacerbations? J Bras Pneumol. 2013;39:257-258.

5. Sasaki T, Nakayama K, Yasuda H, Yoshida M, Asamura T, Ohrui T, Arai H, Araya J, Kuwano K, Yamaya M. HYPERLINK “/ pubmed/19515110"A randomized, single-blind study of lansoprazole for the prevention of exacerbations of chronic obstructive pulmonary disease in older patients. J Am Geriatr Soc. 2009;57:1453-7. 